\title{
The Ambivalent Healthcare Human
}

\author{
Samuel Greenstein ${ }^{1}$
}

Received: 3 March 2021 / Accepted: 13 May 2021 / Published online: 24 May 2021

(C) Academic Psychiatry 2021

I am a human, but I am also a healthcare hero. In the traditional sense, heroes performed great feats and generally had a mythological quality about them, often with a divine connection. This interpretation became conflated with the modern comic superhero-many of whom had some form of an "unnatural" superpower. Today, heroes are recognized as being more human like and allowed to be "flawed." But this does not necessarily stop the preconceptions.

Every day since the pandemic hit New York City, I am reminded that I am a "healthcare hero." I certainly do not feel like one, but it is a ubiquitous sentiment all around me. I either walk past a sign that is "thanking our healthcare heroes," see a picture of a healthcare clinician alongside Batman, Captain America, and Wonder Woman, or I look into a mirror that says something to the extent of, "Here stands a hero." This recognition is humbling and inspiring, but also confusing.

I am a consultation-liaison (CL) psychiatrist at Long Island Jewish Medical Center-one of many hospitals under the Northwell Health system in New York. Officially, we are a 583-bed facility. On our worst day this past spring of 2020, we had over 750 COVID+ patients admitted in our hospital, and a total of 900 admitted patients. As a CL psychiatrist, I had the unique experience of caring for these patients, whether it would be assisting in the treatment for delirium, addressing anxiety related to a patient with COVID-19 who is having low oxygen saturation, or assisting in the medical management of patients with severe and persistent mental illness that now have COVID-19. I also bore witness to what can only be described as madness. It is not hyperbolic to state that there would be 20 or more rapid response pages overhead per day. I heard many stories of how many patients died, and how many phone calls were relayed to family members who were unable to be there during their loved one's final breaths. Several hospitalists told me that the number of deaths they saw in a

Samuel Greenstein

sgreenstei@northwell.edu

1 Donald and Barbara Zucker School of Medicine at Hofstra/ Northwell, Hempstead, NY, USA week was greater than an entire years' worth of treating patients.

When I look back, I did not fully appreciate how enormous the situation was. It was one step in front of the other for me and telling myself to "keep moving forward." Most likely this was a defense mechanism. Because, like many of us, our work life was only half the story. We all had personal lives to attend to, each one unique, some more devastating than others. The fact that our healthcare system did not breakdown is truly a testament to its character and resilience. Healthcare systems and our government either recognized or insinuated that this is a battle - utilizing language such as "redeployment," "frontline," and "war." And while it can be described as heroic, it no doubt took a physical and mental toll on everyone involved. We left those months severely emotionally injured, and now many healthcare colleagues (not just physicians, nurses, and respiratory therapists, but the entire healthcare team) are experiencing post-traumatic stress disorder (PTSD).

Healthcare clinicians, and more notably physicians, breed a type of personality that is perfectionistic, driven, and determined. There is likely an unconscious hero-esque personality that is embedded deep within. These unconscious feelings can to a degree explain why physicians are notorious for not seeking their own personal help. The lack of help seeking partially explains burnout. And now more than ever, burnout, anxiety, depression, and PTSD are staring all of us in the face. How can we possibly go through another surge?

One of the main principles I continue to teach my residents and fellows is that ambivalence, or conflicting feelings, is normal. In fact the urge to resolve such binary or opposite feelings (e.g., love and hate, good and bad, strong and weak) often causes anxiety, irritability, or decreased overall wellbeing. We can be annoyed while at the same time be empathic. We can be scared and anxious but at the same time feel strong. Furthermore, we do not always have to resolve these seemingly conflicting feelings, and acceptance of emotions has been linked with great psychological well-being [1]. Crying is not only ok, but perhaps welcomed. Personally, I felt all of these emotions those months, and cried on two separate occasions. Sometimes I picked myself up, other times my team or 
immediate support picked me up. The truth is that we can ask for help, while at the same time be a hero (in fact many superheroes seem to have imperative sidekicks). We must rely on our team and perhaps form new teams. Stopping the stigma must be more than a hashtag found on social media. We must be there for each other. We must ask for help.

This late December and January, I received my two doses of the COVID-19 vaccine. This momentous occasion happened concurrently during the most recent subsequent surge. And naturally there were many dialectical ambivalent feelings. I was anxious, angry, and frustrated, but also inspired, hopeful, and truly humbled. And once I received the vaccine, I added the feelings of privileged and gratitude. It was important to acknowledge all these feelings rather than feel the need to resolve them. I say this out loud mainly as a way to ground myself, because while cases are starting to decline, the frontline healthcare heroes are still aggressively fighting off the enemy. I know we are not out of the woods yet, and I will likely need my sidekicks.

So yes, we are healthcare heroes. But we are also human. We can be both.

\section{Declarations}

Disclosures The author states that there is no conflict of interest.

\section{Reference}

1. Ford BQ, Lam P, John OP, Mauss IB. The psychological health benefits of accepting negative emotions and thoughts: laboratory, diary, and longitudinal evidence. J Pers Soc Psychol. 2018;115: 1075-92. https://doi.org/10.1037/pspp0000157.

Publisher's Note Springer Nature remains neutral with regard to jurisdictional claims in published maps and institutional affiliations. 